\title{
Correction to: Long noncoding RNA LINC00336 inhibits ferroptosis in lung cancer by functioning as a competing endogenous RNA
}

\author{
Min Wang ${ }^{1,2,3} \cdot$ Chao Mao ${ }^{1,3} \cdot$ Lianlian Ouyang ${ }^{1,3,4} \cdot$ Yating Liu $^{1,3} \cdot$ Weiwei Lai ${ }^{1,3} \cdot$ Na Liu $^{1,3} \cdot$ Ying Shi $^{1,3} \cdot$ Ling Chen $^{1,3}$. \\ Desheng Xiao ${ }^{5}$. Fenglei $\mathrm{Yu}^{6} \cdot \mathrm{Xiang} \mathrm{Wang}^{6} \cdot \mathrm{Hu} \mathrm{Zhou}^{7} \cdot \mathrm{Ya} \mathrm{Cao}^{1,3} \cdot$ Shuang Liu ${ }^{4}$ Q Qin Yan $\mathbb{D}^{8}$. \\ Yongguang Tao $\mathbb{1}^{1,3,6} \cdot$ Bin Zhang $^{2}$
}

Published online: 5 August 2019

(c) The Author(s) 2019

\section{Correction to: Cell Death \& Differentiation \\ https://doi.org/10.1038/s41418-019-0304-y \\ published online 20 February 2019}

Since publication of this article, the authors reported that the names of the corresponding authors had been placed in the wrong order.
The correct order is as follows:

Min Wang, Chao Mao, Lianlian Ouyang, Yating Liu, Weiwei Lai, Na Liu, Ying Shi, Ling Chen, Desheng Xiao, Fenglei Yu, Xiang Wang, Hu Zhou, Ya Cao, Shuang Liu, Qin Yan, Yongguang Tao \& Bin Zhang

This has been corrected in both the PDF and HTML versions of the Article.
These authors contributed equally: Min Wang, Chao Mao

\section{Edited by S. Fulda}

Yongguang Tao

taoyong@csu.edu.cn

$\triangle$ Bin Zhang

coolzhangbin22@163.com

1 Key Laboratory of Carcinogenesis and Cancer Invasion, Ministry of Education, Department of Pathology, Xiangya Hospital, Central South University, Changsha, Hunan 410078, China

2 Department of Histology and Embryology, School of Basic Medicine, Central South University, Changsha, Hunan 410013, China

3 NHC Key Laboratory of Carcinogenesis (Central South University), Cancer Research Institute, Central South University, Changsha, Hunan 410078, China
4 Department of Oncology, Institute of Medical Sciences, Xiangya Hospital, Central South University, Changsha, Hunan 410008, China

5 Department of Pathology, Xiangya Hospital, Central South University, Changsha, Hunan 410008, China

6 Department of Thoracic Surgery, Second Xiangya Hospital, Central South University, Changsha 410011, China

7 Shanghai Institute of Material Medica, Chinese Academy of Sciences (CAS), 555 Zu Chongzhi Road, Zhangjiang Hi-Tech Park, Shanghai 201203, China

8 Department of Pathology, Yale School of Medicine, New Haven, CT 06520, USA 